\title{
Correlação entre avaliação física virtual e de desempenho funcional em mulheres: Um estudo piloto
}

\author{
Correlation between virtual physical assessment and functional performance in women: A pilot \\ study
}

\author{
Marina Andrade Donzeli ${ }^{1, *}$ \\ Lucas Gabriel Coelho Gomes ${ }^{1}$ \\ Eduardo Henrique Pereira Rodrigues ${ }^{1}$ \\ Gustavo de Mello Rodrigues ${ }^{1}$ \\ Dernival Bertoncello ${ }^{1}$ \\ Daniel Ferreira Moreira Lobato ${ }^{1}$
}

\section{Resumo}

Objetivo: Verificar o nível de correlação entre uma avaliação virtual de aptidão física com medidas de desempenho funcional em mulheres ativas. Método: Estudo preliminar, de natureza transversal, cuja amostra foi constituída por 37 mulheres sadias $\left(21,97 \pm 2,60\right.$ anos e $\left.22,86 \pm 3,22 \mathrm{~kg} / \mathrm{m}^{2}\right)$ e ativas $(233,68 \pm 132,06$ minutos de atividade física/semana). A avaliação virtual de aptidão física foi realizada por meio do jogo Nike+ Kinetic Training $\AA$, em ambiente XBOX 360/Kinetic $\AA$, que apresenta um módulo de avaliação do preparo e do condicionamento físico. A avaliação do desempenho funcional foi realizada por meio do shuttlerun (SR) e do six-meter timed hop test (STHT). Os resultados foram correlacionados por meio do índice produto-momento de Pearson, considerando $\alpha=5 \%$. Resultados: Houve correlação moderada entre: o nível de condicionamento físico e o desempenho no $S R(R=-0,42, p=0,009)$; os níveis de preparo $(R=-0,53 ; p<0,001)$ e condicionamento físico $(R=-0,5 ; p=0,01)$ e o desempenho no STHT-membro não-dominante; o nível de condicionamento físico e o desempenho no STHT-membro dominante $(R=-0,4 ; p=0,01)$. Conclusão: Os níveis de condicionamento físico parecem representar de forma mais adequada 0 desempenho funcional do que os de preparo físico. Entretanto, a avaliação virtual utilizada não apresentou elevada correlação com os testes de desempenho funcional.
\end{abstract}

Palavras-chave: terapia de exposição à realidade virtual, desempenho atlético, membro inferior.

\begin{abstract}
Objective: To verify the correlation level between a virtual fitness assessment and functional performance measurements in active women. Method: Preliminary cross-sectional study with 37 healthy $\left(21.97 \pm 2.60\right.$ years and $\left.22.86 \pm 3.22 \mathrm{~kg} / \mathrm{m}^{2}\right)$ and active $(233.7 \pm 132.3$ minutes/week of physical activity) women. Virtual fitness assessment was performed using the Nike+ Kinetic Training $^{\mathrm{TM}}$ game in a XBOX 360/Kinetic ${ }^{\mathrm{TM}}$ environment, which features a physical fitness and a conditioning assessment modules. The functional performance evaluation was performed using the shuttle run (SR) and the six-meter timed hop test (STHT). The correlationswere obtained using the Pearson's product-moment index, considering $\alpha=5 \%$. Results: There was a moderate correlation between: conditioninglevel and SR performance $(R=-0.42, p=0.009)$; physical fitness $(R=-0.53, \quad p<0.001)$ and conditioning $(R=-0.5 ; p=0.01)$ levels and non-dominant SHTH performance; and conditioning level and dominant STHT performance $(R=-0.4 ; p=0.01)$. Conclusion: Conditioning levels seem to represent more appropriately functional performance than physical fitness levels. However, the virtual fitness assessment applied was not highly correlated with functional performance tests.
\end{abstract}

Keywords: virtual reality exposure therapy, athletic performance, lower extremity.
Afiliação dos autores

${ }^{1}$ Universidade Federal do Triângulo Mineiro, Uberaba, Minas Gerais, Brasil.

${ }^{*}$ Autor correspondente

Rua Vigário Carlos, 100, Bloco B, $4^{\circ}$ andar, Sala 406, CEP: 38025-350, Uberaba, Minas Gerais, Brasil. e-mail: marina_donzeli@hotmail.com

Conflito de interesses

Os autores declararam não haver conflito de interesses.

Processo de arbitragem

Recebido: 14/02/2019 Aprovado: 03/03/2019 


\section{Introdução}

O movimento funcional consiste na capacidade de produzir e manter um equilíbrio entre a mobilidade e a estabilidade ao longo da cadeia cinética enquanto executa padrões fundamentais com precisão e eficiência ${ }^{1}$. Requer os seguintes componentes: força muscular, flexibilidade, resistência, coordenação, equilíbrio e eficiência, que são essenciais para o desempenho e habilidades relacionadas ao esporte ${ }^{1,2}$.

A avaliação dos componentes do movimento funcional é necessária e usualmente estimulada no contexto esportivo. Para tanto, são preconizados diversos testes de desempenho físico nas avaliações de força, potência e agilidade durante movimentos multiarticulares ${ }^{3,4}$. Os protocolos de testes mais comuns são escolhidos devido ao seu baixo custo, simplicidade, portabilidade e acessibilidade, destacando, portanto, sua aplicabilidade clínica.

Dentre os testes de desempenho funcional mais utilizados, destacam-se aqueles que avaliam o desempenho por meio de saltos verticais e em distância, utilizando apoio unipodal e bipodal ${ }^{5,6}$, não apenas em indivíduos saudáveis, mas também em populações específicas como pacientes após reconstrução do ligamento cruzado anterior (LCA), visando o retorno seguro à prática esportiva ${ }^{5,7}$. Entretanto, questiona-se se esses testes são realmente bons preditores de lesão ou recidivas, reforçando a utilização de um conjunto maior de avaliações clínicas para melhor identificar possíveis déficits funcionais ${ }^{8}$.

Neste sentido, Okada et al. ${ }^{9}$ propõem uma investigação de desempenho funcional mais abrangente, envolvendo a avaliação da função e resistência muscular do CORE durante os movimentos de flexão, extensão e flexão lateral do tronco, de forma complementar à avaliação dos movimentos dinâmicos. Desse modo, é importante utilizar métodos de avaliação física e de desempenho funcional que avaliem diferentes fatores estruturais e biomecânicos, principalmente para as mulheres, que apresentam um aumento relativo no risco de lesão de 15$16 \%$ quando expostas à prática esportiva em associação com a alta flexibilidade ${ }^{10}$.

Paralelamente aos recursos convencionais utilizados na avaliação funcional e reabilitação, a realidade virtual tem sido considerada como um recurso que promove melhoria da locomoção, da coordenação, da funcionalidade, além de motivar o paciente à prática do exercício ${ }^{11}$. Os jogos denominados exergames (EXGs) têm como objetivo envolver a exploração da movimentação corporal em atividades como correr, pular, abaixar ou deitar, utilizando representações gráficas no ambiente virtual e possibilitando a interação do indivíduo com o aparelho eletrônico, por meio de um sistema de computador que permite um feedback sensorial, de forma visual, auditiva ou tátil $^{12}$.

Dentre os instrumentos utilizados para essa prática, destaca-se o crescente uso do Microsoft Kinect®, que por meio de um sensor de captura de movimentos permite interações do jogador (com o jogo), refletindo em algum tipo de desempenho, que poderia ser mensurado ${ }^{13}$. Assim, aliado ao aspecto motivacional que os EXGs fornecem aos praticantes dessas atividades, sugere-se que 0 uso da realidade virtual deva ser mais estimulado para as avaliações físicas e funcionais, de forma complementar aos testes funcionais comumente utilizados no contexto clínico e esportivo.

A literatura consultada revela estudos que utilizaram a tecnologia de jogos de interação para a reabilitação ou em protocolos de prevenção de lesões, incrementando a resistência da musculatura, aumentando a força muscular e/ou o nível de atividade física ${ }^{14,15}$. Algumas vantagens apontadas consistem em poder controlar o ambiente, neutralizar o impacto dos fatores ambientais sobre a capacidade do indivíduo e, assim, refletir de forma mais adequada a sua aptidão ajustada ao ambiente. Entretanto, destaca-se o reduzido número de estudos ${ }^{16,17}$ que tenham investigado algum aspecto relacionado ao desempenho funcional, com resultados ainda a esclarecer. Portanto, o objetivo do estudo foi verificar o nível de correlação entre uma avaliação virtual de aptidão física com medidas de desempenho funcional em mulheres ativas.

\section{Métodos}

Trata-se de estudo piloto, aprovado pelo Comitê de Ética em Pesquisa da Universidade Federal do Triângulo Mineiro, sob o número $\mathrm{n}^{\circ}$ 2.073.109/2017. Fizeram parte da amostra 37 pessoas do sexo feminino, recrutadas por meio de divulgação do projeto nas dependências da Universidade. Os dados de caracterização das participantes da pesquisa estão apresentados na Tabela 1.

As voluntárias assinaram previamente um Termo de Consentimento Livre e Esclarecido e foram selecionadas por meio de uma entrevista, com questionamentos sobre o nível de atividade física e o estado de saúde. Participaram da amostra apenas mulheres saudáveis e, praticantes de atividades físicas, no mínimo 3x/semana, durante no mínimo 30 minutos/treino ou atividade, com idade entre dezoito e trinta anos. Foram excluídas as candidatas que apresentavam problemas graves de saúde, lesões atuais ou prévias, sinais de inflamação, dor, instabilidade articular nos membros inferiores e a idade não requerida.

Tabela 1

Média e desvio-padrão dos dados antropométricos e demográficos da amostra $(n=37)$.

\begin{tabular}{lc}
\hline \multicolumn{1}{c}{ Variável } & Média \pm Desvio Padrão \\
\hline Idade (anos) & $21,97 \pm 2,60$ \\
Massa corporal $(\mathrm{kg})$ & $60,31 \pm 9,39$ \\
Estatura $(\mathrm{m})$ & $1,62 \pm 0,06$ \\
İndice de massa corporal $\left(\mathrm{kg} / \mathrm{m}^{2}\right)$ & $22,86 \pm 3,22$ \\
Nível de atividade física $(\mathrm{min} / \mathrm{semana})$ & $233,68 \pm 132,06$ \\
\hline
\end{tabular}

\section{Procedimentos}

A avaliação consistiu em um único dia e utilizou-se uma ficha de avaliação física, fitas para demarcação da área nos testes de desempenho funcional, videogame XBOX $360^{\circ}{ }_{\circledast}$ (Microsoft Inc., USA) associado a um sensor Kinetic® (Microsoft Inc., USA) de movimento e televisor de 50 polegadas (AOC International, Taipé, Taiwan), bem como o jogo Nike+ Kinetic Training ${ }^{\circledR}$ (Ubisoft Inc., USA).

\section{Avaliação funcional}

A avaliação do desempenho funcional foi realizada por meio de dois testes funcionais: shuttlerun (SR) e six-meter timed hop test (STHT), considerados como medidas de agilidade ${ }^{18}$.

No teste do $S R$, as voluntárias correram por uma distância de 6,12 metros em deslocamento anterior (em máxima velocidade) e tocaram com o pé uma linha demarcada no solo. A seguir, realizaram uma rápida mudança de direção e retornaram ao ponto inicial de partida, tocando com o pé a linha inicial e repetiram este procedimento por mais uma vez ${ }^{19}$. O teste completo envolveu a distância de 24,4 metros e três mudanças de direção (uma ao final de cada deslocamento), sendo o tempo para a execução do teste cronometrado (Timexmarathon, TimexGroup USA Inc, Middlebury, CT, USA) em segundos (considerando até a casa centesimal) e registrado para as comparações de interesse. Para a análise dos dados foi considerada a média das três tentativas válidas.

No teste do STHT, as voluntárias iniciaram o teste em apoio simples sobre um dos membros inferiores (ordem a ser determinada por sorteio), com o pé imediatamente atrás da linha de partida. Os membros superiores foram posicionados atrás do tronco para evitar qualquer contribuição para o equilíbrio durante a atividade, aumentando a demanda funcional no membro inferior em consideração. As voluntárias foram instruídas a completar a distância definida (6 metros) realizando saltos consecutivos sobre um dos membros inferiores o mais rápido possível e a cruzar a linha de chegada sem desacelerar em qualquer momento do teste ${ }^{20}$, sendo o tempo para execução do teste cronometrado.

Os testes foram repetidos quando as voluntárias realizaram algum procedimento incompatível com as instruções do teste, quando utilizaram seus membros superiores como uma estratégia de propulsão ou se houve perda de equilíbrio durante o teste. Três tentativas válidas de cada teste foram realizadas para cada membro inferior. Para a análise dos dados foi considerada a média das três tentativas válidas.

\section{Avaliação do nível de preparo físico e de condicionamento físico}

Para avaliação do nível de preparo físico e de condicionamento físico foi utilizado o jogo NIKE+ Kinect training ${ }^{\circledR}$ em ambiente XBOX 360\%/Microsoft Kinetic ${ }^{\circledR}$, 
conectado a uma televisão. Este jogo ocorre baseado na leitura realizada pelo Kinect $\AA^{\circledR}$ acerca do movimento executado, levando em conta todos os pontos articulares, e convertendo essa leitura em algum tipo de desempenho (Figura 1).

Inicialmente o jogador informou seus dados pessoais, como massa corporal, estatura, idade e sexo. Essas informações são utilizadas pelo software para analisar o nível de preparo físico e de condicionamento físico, após a finalização de uma série sistematizada de exercícios para avaliar a amplitude de movimento (mobilidade) e condicionamento físico gera (preparo físico e condicionamento físico), em seu modo de avaliação ("Desafio físico").

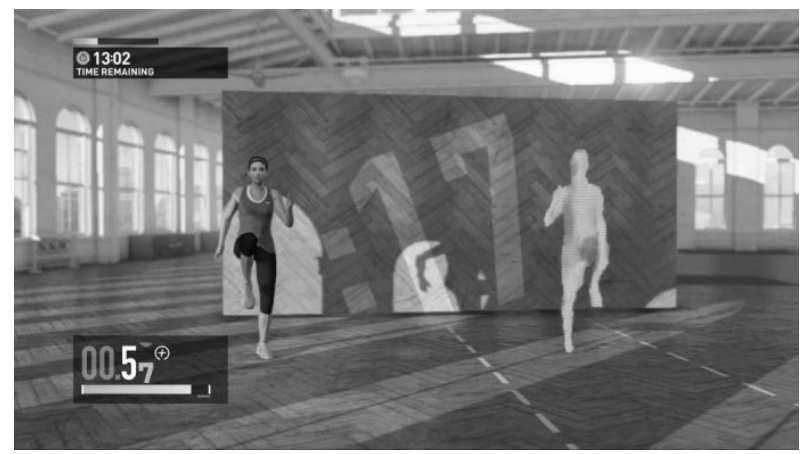

Figura 1. Tela do jogo Nike+ Kinetic Training. Fonte: http://www.nike.com

Na primeira parte da avaliação, correspondente à avaliação da mobilidade, o jogo utilizou de sete movimentos padronizados envolvendo equilíbrio, mobilidade e flexibilidade, seguindo os movimentos avaliativos do método Functional Movement Systems (método FMS): 1) agachamento profundo, 2) passo sobre obstáculos, 3) avanço alinhado, 4) mobilidade dos ombros, 5) levantamento ativo de pernas, 6) flexão com estabilidade do tronco e 7) estabilidade rotacional.

Na segunda parte da avaliação, correspondente à avaliação do preparo físico e do condicionamento físico, o jogo utilizou de seis testes físicos para a avaliação da agilidade, força e resistência: 1) salto e parada balanceados, 2) salto com agachamento, 3) velocidade, reflexos e agilidade, 4) agachamento unilateral, 5) flexão de braços e 6) flexão rápida de joelhos. Ao final desses testes, o jogo calculou, de acordo com o desempenho do praticante e com os dados antropométricos e demográficos informados, um escore correspondente ao nível atual de preparo físico e um escore correspondente ao nível atual de condicionamento físico.

\section{Análise estatística}

Para a análise dos dados foi utilizado o programa Statistica 5.0 for Windows (StatSoft). As medidas referentes ao nível de preparo e condicionamento físico, bem como de desempenho funcional foram analisadas inicialmente através da estatística descritiva (média e desvio-padrão) e, de acordo com as características das distribuições de seus dados (verificada pelo teste de Shapiro Wilks), correlacionadas pelo índice produtomomento de Pearson, considerando um nível de significância de $5 \%$.

\section{Resultados}

Os dados referentes ao nível de preparo e condicionamento físico obtido na avaliação virtual de desempenho, bem como os resultados dos testes de desempenho funcional estão apresentados na Tabela 2.

Tabela 2

Média e desvio-padrão dos dados referentes ao nível de preparo e condicionamento físico e ao desempenho funcional $(n=37)$

\begin{tabular}{lc}
\multicolumn{1}{c}{ Testes } & Média \pm Desvio Padrão \\
\hline Preparo físico (pontos) & $45,32 \pm 10,36$ \\
Condicionamento físico (pontos) & $49,03 \pm 7,40$ \\
SR (s) & $9,36 \pm 0,53$ \\
STHT - MDOM (s) & $2,73 \pm 0,41$ \\
STHT - MNDOM (s) & $2,82 \pm 0,48$ \\
\hline
\end{tabular}

$\mathrm{SR}=$ shuttle run; $\mathrm{STHT}=$ six-meter timed hop test

$\mathrm{MDOM}=$ membro dominante; MNDOM = membro não-dominante
A partir desses resultados, a Tabela 3 apresenta o nível de correlação verificado entre os testes de desempenho virtual e os testes funcionais utilizados.

Tabela 3

Nível de correlação (e significância estatística) entre as variáveis de preparo e condicionamento físico e as medidas de desempenho funcional $(n=37)$

\begin{tabular}{lcccc}
\hline & \multicolumn{2}{c}{ Preparo físico } & \multicolumn{2}{c}{ Condicionamento físico } \\
\hline & $\mathrm{R}$ & $p$-valor & $\mathrm{R}$ & $p$-valor \\
SR & $-0,28$ & 0,09 & $-0,42$ & 0,009 \\
STHT-MDOM & $-0,3$ & 0,08 & $-0,4$ & 0,01 \\
SHTH-MNDOM & $-0,53$ & 0,0007 & $-0,5$ & 0,01 \\
\hline \multicolumn{2}{l}{ SR= shuttle run; STHT $=$ six-meter timed hop test }
\end{tabular}

$\mathrm{MDOM}=$ membro dominante; $\mathrm{MNDOM}=$ membro não-dominante

Houve correlação moderada entre: o nível de condicionamento físico e o desempenho no $S R$; os níveis de preparo e condicionamento físico e o desempenho no STHTmembro não-dominante; o nível de condicionamento físico e o desempenho no STHT- membro dominante. Não houve correlação entre o nível de preparo físico e os desempenhos no $S R$ e no STHT- membro dominante.

\section{Discussão}

O presente estudo buscou verificar o nível de correlação entre uma avaliação virtual de aptidão física por meio do jogo Nike+Kinetic Training ${ }^{\circledR}$ com medidas de desempenho funcional. Foi verificado que o nível de condicionamento físico apresentou melhor correlação com as medidas de desempenho funcional do que o nível de preparo físico. Entretanto, as correlações verificadas foram no máximo moderadas.

Esses resultados apresentam concordância indireta com Brito et al. ${ }^{17}$, que verificaram a existência de uma correlação moderada entre o desempenho no jogo Nike +Kinetic Training $\mathbb{B}$ com as medidas de movimento avaliadas pelo método FMS. Embora não tenham avaliado o desempenho funcional por meio de testes de velocidade e agilidade, como no presente estudo, os autores apoiam o uso do jogo como método de avaliação funcional e sustentam a condição de equivalência que a realidade virtual pode apresentar em relação ao desempenho em testes funcionais clínicos.

Evidências apontam que utilizando uma bateria de testes funcionais é possível predizer se há a necessidade de reabilitação adicional antes de voltar ao esporte no caso de cirurgia de reconstrução de LCA e utilizam testes de saltos, distâncias e saltos laterais para avaliar a estabilidade postural, utilizando apoio unipodal e bipodal ${ }^{5,7}$, porém a qualidade do movimento não é avaliada. Sendo assim, faz-se necessário um refinamento deste tipo de avaliação funcional. Ao adicionar novas tecnologias como a realidade virtual e seu amplo aspecto avaliativo, sugere-se a possibilidade de melhor identificar déficits funcionais e desequilíbrios musculares.

Neste sentido, na primeira parte da avaliação virtual proposta no presente estudo, o jogo utilizou do método FMS para avaliar a mobilidade com sete movimentos padronizados envolvendo equilíbrio, mobilidade e flexibilidade. Lopes et al. ${ }^{21}$ correlacionaram a avaliação FMS com as lesões em atletas de jiu jitsu, descobrindo que os locais mais acometidos foram os joelhos, coluna e ombros, e utilizaram a análise biomecânica do movimento para realizar um posterior trabalho preventivo. Além disso, Boyle ${ }^{22}$ sugere a influência da mobilidade no movimento e como isso pode gerar compensações e, consequentemente, dores e lesões, apontando a avaliação do movimento como um fator determinante na prevenção e reabilitação.

Os EXGs já são muito utilizados em tratamentos de reabilitação e para promover um estilo de vida ativo ${ }^{15,18}$. O presente estudo, embora de caráter piloto, adiciona uma outra aplicabilidade a eles, relacionada à avaliação das capacidades físicas e habilidades motoras, envolvendo aspectos relacionados à função muscular. Apresentam-se como uma ferramenta que usa movimentos imprevistos e de agilidade sempre com um retorno verbal e visual ${ }^{23}$, sendo importante para analisar o real condicionamento físico do indivíduo.

Além disso, uma revisão com 27 estudos mostra a forte correlação entre os EXGse o aumento do gasto de energia (até $300 \%$ acima dos níveis de repouso), afirmando que a maioria dos videogames ativos testados atingiu níveis de atividade física de intensidade moderada, que atendem às diretrizes do Colégio 
Americano de Medicina Esportiva para saúde e condicionamento físico ${ }^{14}$. Especificamente em relação ao jogo Nike+Kinetic Training $\AA$, Brito et al. ${ }^{17}$ verificaram moderada correlação entre o desempenho no jogo com a Escala de Percepção de Esforço e também demonstraram que, em relação à intensidade do teste, embora haja variabilidade do resultado, o valor médio classifica a atividade como moderada. Em virtude da dificuldade de encontrar mais estudos com o uso da realidade virtual como meio de avaliação funcional, não foi possível estabelecer valores padronizados por dados, e a consequente comparação de resultados para confrontar com a presente investigação. Este fato chama atenção à importância do presente estudo.

A literatura nos traz correlações de avaliação de força e desempenho funcional, principalmente em grupos específicos. Gadelha et al. ${ }^{24}$ relatam associação positiva entre a força muscular e o desempenho funcional de pessoas idosas. Os resultados do presente estudo, embora com diferenças metodológicas, apontam para associações de mesma tendência. Entretanto, ao considerarmos que o jogo utilizado avalia outras valências físicas (como a força e resistência muscular, tempo de reação e equilíbrio), além dos aspectos de velocidade, agilidade e explosão (principais valências avaliadas no SR e STHT), podemos sugerir que este tenha sido um fator limitante em gerar maior associação entre as medidas.

Outro ponto relevante está relacionado ao uso de testes funcionais de característica bilateral ou unilateral. Myer et al. ${ }^{25}$ concluíram que, em atletas que fizeram reconstrução do LCA, os déficits unilaterais presentes podem não ser evidentes durante 0 desempenho bipodal ou durante versões modificadas de atividades de desempenho de duplo apoio. Assim, sugerem que o isolamento do membro envolvido, com tarefas de salto unilateral, deve ser usado para identificar déficits no desempenho. Esta pode ser uma explicação parcial para a ausência de correlações fortes entre as medidas de desempenho virtual e real (por saltos), visto que a primeira abordou, em sua maioria, testes realizados em duplo apoio, enquanto o STHT é um teste exclusivamente unipodal.

Além disso, verifica-se que os testes de salto, de forma isolada, não fornecem informações clínicas suficientes para tomada de decisão sobre a força muscular dos membros inferiores, mas durante os saltos o posicionamento do tronco influencia na ativação dos músculos do quadril ${ }^{26-28}$. Desse modo, é importante a análise corporal completa, como realizada na avaliação virtual, que o sensor é sensível a qualquer movimento e consegue captar esses posicionamentos, estimulando uma maior utilização da musculatura ${ }^{29}$.

As investigações para validar os EXGs nos últimos anos têm aumentado, principalmente utilizando o Kinect®, em que os usuários podem controlar e interagir com os jogos por meio do movimento corporal, porém o número de estudos ainda não permite estabelecer um consenso ${ }^{30}$. Deste modo, destaca-se como limitação nesta temática o reduzido número de trabalhos com a utilização de estudos comparativos utilizando 0 Xbox $360 \%$ Kinect ${ }^{\circledR}$ como meio de avaliação funcional, o que dificulta uma discussão mais aprofundada sobre o tema.

\section{Conclusão}

Os níveis de condicionamento físico parecem representar de forma mais adequada o desempenho funcional do que os de preparo físico. Entretanto, a avaliação virtual utilizada não apresentou elevada correlação com os testes de desempenho funcional.

\section{Financiamento}

Coordenação de Aperfeiçoamento de Pessoal de Ensino Superior - CAPES (bolsa DS/CAPES - Mestrado); Fundação de Amparo à Pesquisa do Estado de Minas Gerais - FAPEMIG (bolsa de Iniciação Científica).

\section{Referências}

1. Mills JD, Taunton JE, Mills WA. The effect of a 10-week training regimen on lumbo-pelvic stability and athletic performance in female athletes: a randomized-controlled trial. Phys Ther Sport. 2005; 6:60-66.

2. Cook G. Baseline sports-fitness testing. In: High Performance Sports Conditioning. B. Foran, ed. Champaign, IL: Human Kinetics Inc; 2001.
3. Kroman SL, Roos EM, Bennell KL, Hinman, RS, Dobson F. Measurement properties of performance-based outcome measures to assess physical function in young and middle-aged people known to be at high risk of hip and/or knee osteoarthritis: a systematic review. Osteoarthr. Cartil. 2014; 22(1):26-39.

4. Hegedus EJ, McDonough S, Bleakley C, Cook CE, Baxter GD. Clinicianfriendly lower extremity physical performance measures in athletes: a systematic review of measurement properties and correlation with injury, part 1. The tests for knee function including the hop tests. Br J Sports Med. 2015; 49(10):642-648.

5. Hildebrandt C, Müller L, Zisch B, Huber R, Fink C, Raschner C. Functional assessments for decision-making regarding return to sports following $\mathrm{ACL}$ reconstruction. Part I: development of a new test battery. Knee Surg Sports TraumatolArthrosc. 2015; 23(5):1273-81.

6. Abrams GD, Harris JD, Gupta AK, McCormick FM, Bush-Joseph CA, Verma NN, et al. Functional Performance Testing After Anterior Cruciate Ligament Reconstruction: A Systematic Review. Orthop J Sports Med. 2014; 2(1):2325967113518305.

7. Paterno MV, Rauh MJ, Schmitt LC, Ford KR, Hewett TE. Incidence of contralateral and ipsilateral anterior cruciate ligament (ACL) injury after primary ACL reconstruction and return to sport. Clin J Sport Med. 2012 22(2):116-21

8. Hegedus EJ, McDonough SM, Bleakley C, Baxter D, Cook CE. Clinicianfriendly lower extremity physical performance tests in athletes: a systematic review of measurement properties and correlation with injury. Part 2--the tests for the hip, thigh, foot and ankle including the star excursion balance test. Br J Sports Med. 2015; 49:649-56.

9. Okada T, Huxel KC, Nesser TW. Relationship between core stability, functional movement, and performance. J StrengthCond Res. 2011 25(1):252-261.

10. Trudelle-Jackson E, Leonard D, Morrow JR. Musculoskeletal Risk Factors as Predictors of Injury in Community-Dwelling Women. Med Sci Sports Exerc. 2014; 46(9):1752-7.

11. Merians AS, Jack D, Boian R, Tremaine M, Burdea GC, Adamovich SV, et al. Virtual reality-augmented rehabilitation for patients following stroke. Phys Ther. 2002; 82(9):898-915.

12. Mumford N, Wilson PH. Virtual reality in acquired brain injury upper limb rehabilitation: evidence-based evaluation of clinical research. Brain Inj. 2009; 23(3):179-91.

13. Han J, Shao L, Xu D, Shotton J. Enhanced Computer Vision With Microsoft Kinect Sensor: A Review. IEEE Trans Cybern. 2013; 43(5):1318-34.

14. Siriphorn A, Chamonchant D. Wii balance board exercise improves balance and lower limb muscle strength of overweight young adults. J Phys Ther Sci. 2015; 27(1):41-6.

15. Park J, Lee D, Lee S. Effect of Virtual Reality Exercise Using the Nintendo Wii Fit on Muscle Activities of the Trunk and Lower Extremities of Normal Adults. J Phys Ther Sci. 2014; 26(2):271-3.

16. Estevam DO, de Oliveira ML, da Silva ML, Carvalho LC, Lobato DFM Efeitos do resfriamento e aquecimento articular no desempenho funcional do ombro. RevBrasMed Esporte. 2015; 21(3):168-172.

17. Brito EN, Silva BKR, Quaresma FRP, da Silva Maciel E. Avaliação funcional por meio de exergame. Revista Brasileira de Prescrição e Fisiologia do Exercício. 2018; 12(76):541-546.

18. Keays SL, Bullock-Saxton JE, Newcombe P, Keays AC. The relationship between knee strength and functional stability before and after anterior cruciate ligament reconstruction. J Orthop Res. 2003;21(2):231-7.

19. Lephart SM, Kocher MS, Harner CD, Fu FH. Quadriceps strength and functional capacity after anterior cruciate ligament reconstruction. Patellar tendon autograft versus allograft. Am J Sports Med. 1993;21(5):738-43.

20. Baldon R de M, Lobato DFM, Carvalho LP, Wun PYL, Santiago PRP, Serrão FV. Effect of functional stabilization training on lower limb biomechanics in women. Med Sci Sports Exerc. 2012; 44(1):135-45.

21. Lopes OPS, Alves LDB, Falconeri PN, Neto PDSP, de Souza SFM. Prevalência de lesões e avaliação funcional do movimento entre praticantes de Jiu Jitsu. Motricidade. 2018; 14(1): 365-375.

22. Boyle M. Advances in Functional Training: Training Techniques for Coaches, Personal Trainers and Athletes. England: Lotus Publishing 2011.

23. Myer GD, Ford KR, Brent JL, Hewett TE. An integrated approach to change the outcome part II: targeted neuromuscular training techniques to reduce identified ACL injury risk factors. J Strength Cond Res. 2012; 26(8):2272-92.

24. Gadelha AB, Dutra MT, de Oliveira RJ, Safons MP, Lima RM. Associação entre força, sarcopenia e obesidade sarcopênica com o desempenho funcional de idosas. Motricidade. 2014; 10(3):31-39.

25. Myer GD, Schmitt LC, Brent JL, Ford KR, Barber Foss KD, Scherer BJ, et al. Utilization of modified NFL combine testing to identify functional deficits in athletes following ACL reconstruction. J Orthop Sports Phys Ther. 2011; 41(6):377-87.

26. Kollock R, Van Lunen BL, Ringleb SI, Oñate JA. Measures of functional performance and their association with hip and thigh strength. J AthlTrain 2015; 50(1):14-22.

27. Ford KR, Nguyen A-D, Dischiavi SL, Hegedus EJ, Zuk EF, Taylor JB. An evidence-based review of hip-focused neuromuscular exercise interventions to address dynamic lower extremity valgus. J Sports Med. 2015; 6:291-303.

28. Powers CM. The influence of altered lower-extremity kinematics on patella femoral joint dysfunction: a theoretical perspective. J Orthop Sports Phys Ther. 2003; 33(11):639-46

29. Gomes J, Melo R, Oliveira S, Costa M. Exergames podem ser uma ferramenta para acréscimo de atividade física e melhora do condicionamento físico? Rev. Bras. Ativ. Fís. Saúde. 2015; 20(3):232-232.

30. Kamel Boulos, MN. Xbox 360 Kinect exergames for health. Games for Health. 2012; 1(5):326-330. 